certain evidence, it must be contended that there is no justiflcation for the conclusion that sporadic chondrodystrophy of chicken embryos, let alone the corresponding abnormality in man, is due to or in any wsy affected by disturbances of an endocrine nature. One argument against such a conclusion is the fact that sporadic chondrodystrophy of chicken embryos can already be observed at the very stages a which Studitsky made his pituitary implants. It must be questioned also if evidence, such as has been presented by Studitsky, ever justifles the inference that the natural course of events has been duplicated. In experiments which will be described elsewhere I have found that a very high incidence of micromelia can be induced in chicken embryos by injecting, as early as the beginning of the fourth day of development, two units of insulin into the yolk the fourth day of development, two units of insulin into the yolk of eggs. It would be absurd to interpret these results as indicating the involvement of the pancreas or any other endocrine gland in the causation of micromelia. Studitsky's experiments may demonstrate that disproportionate skeletal growth can be imposed at relatively late stages of development, but they certainly do not p

Storrs Agricultural Experiment Station,

WALTER LANDAUER University of Connecticut,

$$
\text { May } 15 .
$$

'Studitsky, A. N., Nature, 157, 427 (1946).

Studitsky, A. N., Bulletin de l'Académie des Sciences de l'URSS, Classe des sciences biologiques 1939, pp. 457-468.

- Studitsky, A. N., C.R. (Doklady) Acad. Sci. l'URSS., 43, 391 (1944).

- Landauer, W., Z. Mikr-Anat. Forsch., 25, 115 (1931).

- Hamburger, v., Phys. Zool., 14, 355 (1941).

' Rudnick, D., $\dot{J}^{\prime}$. Exper. Zool.', 100, 1 (1945).

- Pighini, G. "Biochimica e Terapia Sperimentale", 24 (1937).

\section{Hydrated Cellulose from Jute Fibre}

Ir is well known that the change in the structure of cellulose in ramie produced by the action of caustic soda solution and by subsequent treatment with water depends largely on the concentration of the alkali used, the temperature and the magnitude of tension to which the flbre is subjected during the treatment. Hess and Trogus ${ }^{1}$ showed that if unstretched ramie is treated with $12 \cdot 5-18$ per cent sodium hydroxide solution and afterwards washed with water, the product gives the X-ray pattern of hydrated cellulose. Sakurada and Hutino later pointed out that unstretched ramie treated with 16 per cent sodium hydroxide solution and washed with water for five minute gives in the moist state a structure different from that of hydrated cellulose, and this product in the moist state was designated as 'water water cellulose is converted to hydrated cellulose.

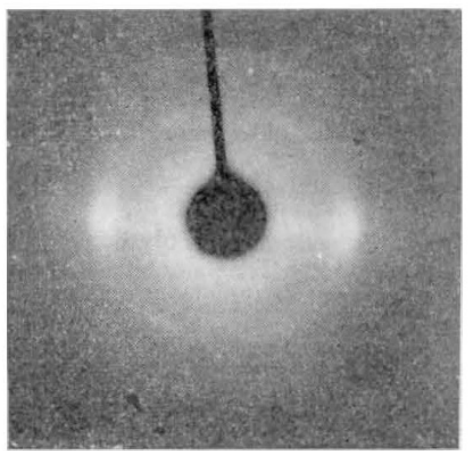

In the course of our work under the scheme on the $\mathrm{X}$-ray analysis of jute fibre financed by the Indian Central Jute Committee, it has of jute fibre financed by the Indian Central Jute Committee, it has been observed that unstretched raw jute for hour, then washed with cent sodium hydroxide solution for half an hour, in free air at room water at about $35^{\circ} \mathrm{C}$. for ten minutes and dried in free air at room temperature for three days, gives a diffraction pattern which is diferent from that due either to water cellulose or to ordinary hydrated cellulose. The spacings of the planes giving intense reflexions in the equatorial line and the dimensions of the mit cell of this new hydrated cellulose obtained from raw jute flbre are given in the accompanying
table along with those for water cellulose and ordinary hydrated cellulose.

\begin{tabular}{|c|c|c|c|}
\hline & Water cellulose & $\begin{array}{l}\text { Ordinary hydrated } \\
\text { cellulose }\end{array}$ & $\begin{array}{l}\text { Hydrated cellulose } \\
\text { from jute }\end{array}$ \\
\hline $\begin{array}{l}A_{1} \\
A_{1} \\
A_{s} \\
a \\
b \\
c \\
\beta\end{array}$ & 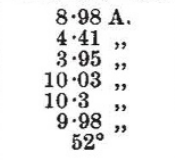 & $\begin{array}{r}7 \cdot 32 \mathrm{~A} . \\
4 \cdot 45, \\
4 \cdot 03, \\
8 \cdot 14, \\
10 \cdot 30 " \\
9 \cdot 14 ” \\
62^{\circ} ",\end{array}$ & 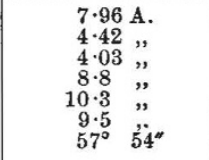 \\
\hline
\end{tabular}

The pattern obtained shows sharp but elongated reflexions indicating the increase in the size of the micelles but deterioration of their orientation along the flbre axis. The physical properties of this hydrated cellulose appear to be similar to those of coarse wool and superior to those of hydrated cellulose obtained from jute flbre with 12 per cent caustic soda. The stability of its structure under different physical conditions is being investigated.

We are indebted to Prof. M. N. Saha for his interest in the work and to the Indian Central Jute Committee for the flnancial help.

Palit Laboratory,

S. C. SIRKAR

University of Calcutta.

May 10.

Z. Chem., B, 11, 381 (1931).

Koll. Z., 77, 346 (1936)

\section{Zero Point Diffusion in Liquid Helium II}

THE fundamental analogy between superconductivity and the $\lambda$-phenomenon of liquid helium to which we directed attention a few years ago ${ }^{1}$ is based on the observation of frictionless transport and on the fact that the superconductive electrons as well as the superfluid helium atoms have zero thermal energy even at finite temperatures ${ }^{2}$. In view of this fact, it has been suggested by one of $\mathrm{us}^{3}$ that the momentum of frictionless transport may be derived from zero point energy and that this transport may even take place in the absence of an external accelerating potential. Indeed this process seems to fit the case of a superconductor in which a flow of electrons occurs under zero electromotive force. On the other hand, it has generally been assumed up to now that in the frictionless transfer of helium II along a surface film ${ }^{4}$ helium atoms are accelerated by the gravitational potential; an explanation which has for some time appeared doubtful to us ${ }^{5}$.

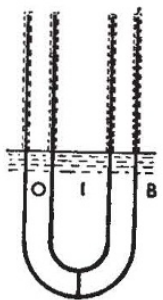

(a)

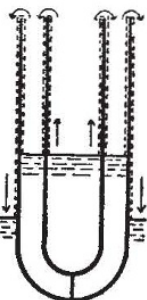

(b)

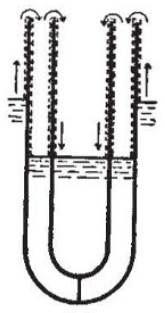

(c)
TRANSFER OF LIQUID HELIUM II UNDER ZERO GRAVITATIONAL POTENTIAL. (a) STATE OF EQUILIBRIUM, (b) BEAKER EMPTYING AND (c) BEAKER FILLING. THE ARROWS INDICATE THE DIRECTION OF FILM TRANSFER

We have now carried out an experiment which demonstrates that this transfer takes place even without gravitational acceleration. A beaker consisting of two concentric vessels was suspended in a bath of liquid helium II. Then the state of equilibrium is the one in which the levels in the inner vessel $(I)$, the outer vessel $(O)$ and to a higher position, $(I)$ and $(O)$ are emptied by surface transfer to a higher position, $(I)$ and $(O)$ are emptied by surface transfer pected rate but without a separation of the levels in $(I)$ and $(O)$. pected rate but without a separation of the levels in $(I)$ and $(O)$. Thus, while helium is transferred from $(O)$ to $(B)$ under a potentia drop, the transfer between $(I)$ and $(O)$ takes place under zero potentia from the observed rate of transfer as well as from considerations from the observed rimensions of the beaker the minimum average arising out of the dimensions of the beaker, the minimum average velocity of the helium atoms flowing from ( $T$ into (O) could be calculated to be about $18 \mathrm{~cm}$./ $/ \mathrm{sec}$. of at least $1.6 \mathrm{~mm}$. between $(I)$ and $(O)$ would have been necessary in order to produce this velocity by gravitational acceleration. Our of the levels in $(I)$ and $(O)$ due to surface tension, showed that no change in the relative position of the levels greater than $\pm 0.1 \mathrm{~mm}$. (our limit of accuracy) took place. The levels in $(I)$ and $(O)$ also stayed together when the flow was reversed by setting the bath leve. above that in $(I)$ and $(O)$. Finally, when the level in $(I)$ was raised above that in (O) by flling it from the bath, it afterwards adjusted itself again to equal height with the level in $(0)$.

Our experimental arrangement is a close analogue to an electric circuit containing a superconductor. In such a circuit the current has to be limited by a resistance so as not to exceed the threshold value. Only if this condition is fulfllled will a super-current under zero eleotrical potential be established. In the beaker the place of this resistance is taken by the diameter of the outer vessel, which limits the rate of helium transfer in the whole arrangement and thereby permits ta flow from $(I)$ to $(O)$ without gravitational acceleration. A more thorough discussion of the experiment has to be left to a detailed publication. However, the result seems to leave no doubt that it is not the gravitational fleld of force which causes the frictionless transfer of liquid helium II. Seen in conjunction with the fact that the superfluid helium atoms have zero thermal energy, the result appears to strengthen our supposition that the phenomena of frictionless transport are direct manifestations of a diffusion of superfuid particles under zero point momentum ${ }^{6}$. Experiments are in preparation for the observation of other effects predicted on this basis ${ }^{3}$, the most striking of which would be isothermal heat conduction and a persistent mass flow in liquid helium II. 